\title{
Fruit and vegetable consumption and risk of depression: accumulative evidence from an updated systematic review and meta-analysis of epidemiological studies
}

\author{
Faezeh Saghafian ${ }^{1,2} \dagger$, Hanieh Malmir ${ }^{1,2} \dagger$, Parvane Saneei ${ }^{3}$, Alireza Milajerdi ${ }^{1,2}$, Bagher Larijani ${ }^{4}$ and \\ Ahmad Esmaillzadeh ${ }^{2,3,5 *}$ \\ ${ }^{1}$ Students' Scientific Research Center, Tehran University of Medical Sciences, PO Box 1417755331, Tehran, Iran \\ ${ }^{2}$ Department of Community Nutrition, School of Nutritional Sciences and Dietetics, Tebran University of Medical Sciences, \\ PO Box 141556117, Tebran, Iran \\ ${ }^{3}$ Department of Community Nutrition, School of Nutrition and Food Science, Food Security Research Center, Isfahan \\ University of Medical Sciences, PO Box 8174673461, Isfahan, Iran \\ ${ }^{4}$ Endocrinology and Metabolism Research Center, Endocrinology and Metabolism Clinical Sciences Institute, Tebran \\ University of Medical Sciences, PO Box 1414413137, Tebran, Iran \\ ${ }^{5}$ Obesity and Eating Habits Research Center, Endocrinology and Metabolism Molecular - Cellular Sciences Institute, \\ Tebran University of Medical Sciences, PO Box 1411713137, Tebran, Iran
}

(Submitted 18 January 2017 - Final revision received 4 January 2018 - Accepted 21 February 2018)

\section{Abstract}

Findings from observational studies investigating the association between fruit and vegetable consumption and risk of depression were inconsistent. We conducted a systematic review and meta-analysis to summarise available data on the association between fruit and vegetable intake and depression. A systematic literature search of relevant reports published in Medline/PubMed, ISI (Web of Science), SCOPUS and Google Scholar until Oct 2017 was conducted. Data from 27 publications (sixteen cross-sectional, nine cohort and two case-control studies) on fruit, vegetables and/or total fruit and vegetable consumption in relation to depression were included in the systematic review. A total of eighteen studies that reported relative risks (RR), hazard ratios or OR for the relationship were included in the meta-analysis. The pooled RR for depression in the highest $v$. the lowest category of fruit intake was 0.83 (95\% CI $0.71,0.98)$ in cohort studies and 0.76 (95\% CI $0.63,0.92)$ in cross-sectional studies. Consumption of vegetables was also associated with a $14 \%$ lower risk of depression (overall RR $=0.86 ; 95 \% \mathrm{CI} 0 \cdot 75,0 \cdot 98$ ) in cohort studies and a $25 \%$ lower risk of depression (overall $\mathrm{RR}=0.75 ; 95 \% \mathrm{CI} 0.62,0.91$ ) in cross-sectional studies. Moreover, an inverse significant association was observed between intake of total fruit and vegetables and risk of depression (overall RR $=0.80$; 95\% CI 0.65 , 0.98) in cross-sectional studies. In a non-linear dose-response association, we failed to find any significant association between fruit or vegetable intake and risk of depression (fruit (cross-sectional studies): $P_{\text {non-linearty }}=0 \cdot 12$; vegetables (cross-sectional studies): $P_{\text {non-linearty }}<0 \cdot 001$; (cohort studies) $\left.P_{\text {non-linearty }}=0.97\right)$. Meta-regression of included observational studies revealed an inverse linear association between fruit or vegetable intake and risk of depression, such that every 100-g increased intake of fruit was associated with a $3 \%$ reduced risk of depression in cohort studies $(\mathrm{RR}=0.97 ; 95 \% \mathrm{CI} 0.95,0.99)$. With regard to vegetable consumption, every 100 -g increase in intake was associated with a $3 \%$ reduced risk of depression in cohort studies ( $\mathrm{RR}=0.97 ; 95 \% \mathrm{CI} 0.95,0.98)$ and $5 \%$ reduced odds in cross-sectional studies (RR $=0.95 ; 95 \% \mathrm{CI} 0.91,0.98)$. This meta-analysis of observational studies provides further evidence that fruit and vegetable intake was protectively associated with depression. This finding supports the current recommendation of increasing fruit and vegetable intake to improve mental health.

Key words: Fruit: Vegetables: Depression: Anxiety: Meta-analyses

The common mental disorders, depression and anxiety, are major public health problems across the globe ${ }^{(1)}$. Depression affects 350 million people worldwide and women are, on average, 1.7 times more likely to have depression than men ${ }^{(2)}$. It is estimated that depression is responsible for $50-70 \%$ of suicides. The WHO predicts that depression will become the second most prevalent disorder (after ischaemic heart disease) by the year $2020^{(3)}$. Anxiety has received special attention during the last couple of decades, owing to its high prevalence and its association with chronic disorders, mainly $\mathrm{CHD}^{(4)}$.

Abbreviation: RR, relative risk.

* Corresponding author: A. Esmaillzadeh, fax: +98 21 88984861, email a-esmaillzadeh@sina.tums.ac.ir

$\dagger$ These authors contributed equally to the manuscript. 
These disorders are considered the main global causes of disability-adjusted life years and economic costs ${ }^{(5,6)}$.

Recent data have highlighted the contribution of modifiable lifestyle behaviours, such as physical inactivity and smoking, to the development of common mental disorders ${ }^{(7)}$. In addition, the relationship between nutrition and depressive disorders has become of increasing interest in recent years ${ }^{(7)}$. Whereas the role of diet in the prevention of other non-communicable diseases, such as CVD, has been widely investigated in the past 50 years, the relationship between diet and mental disorders is so far a novel and interesting field ${ }^{(8)}$. Most previous investigations have focused on the association of depression with specific nutrients, foods and dietary patterns ${ }^{(9)}$. Several studies have suggested that diet quality, for which fruit and vegetable intake is a simple indicator ${ }^{(5)}$, was related to depression in both adults $^{(10,11)}$ and adolescents ${ }^{(12,13)}$.

Findings from earlier observational studies that investigated the association between fruit and vegetable consumption and risk of depression or anxiety were inconsistent. Some investigations have shown a significant association between fruit and vegetable intake and depression ${ }^{(10,14)}$, but other studies found no significant associations ${ }^{(15-17)}$. Although some studies reached significant associations between consumption of fruit and depression, such associations were not significant for vegetable intake ${ }^{(18,19)}$. Some others have reported no significant association between fruit intake and depression ${ }^{(5,16,20,21)}$, but found significant relations with consumption of vegetables ${ }^{(20,21)}$. In addition, there has been a sex difference in the associations. Some investigations have reported a protective association in women $^{(22)}$ or in both sexes ${ }^{(14)}$, and others have reported no association between fruit intake and depression in men or women ${ }^{23)}$. In addition, most studies have reported associations between fruit and vegetable intake and depression, but the linkage with anxiety is less studied ${ }^{(5)}$. A most recent meta-analysis showed that fruit and vegetable intake might be inversely associated with the risk of depression ${ }^{(24)}$; however, the findings might be misleading owing to the lack of inclusion of several published studies in the field ${ }^{(10,14,25-30)}$; also, because of the use of non-appropriate statistical methods, such as combining adjusted and non-adjusted relative risks (RR), incomplete RR extracting and not performing the trim and fill method to identify the effect of unpublished results, their findings might be biased $^{(26)}$. We aimed to conduct a comprehensive systematic review and meta-analysis to summarise available data on the association between fruit and vegetable intake, depression and/ or anxiety.

\section{Methods}

\section{Search strategy}

A computerised search strategy was implemented until Oct 2017 using Pubmed, ISI (Web of Science), SCOPUS, Embase and Google Scholar. The following key words, including those from the medical subject headings (MeSH) database, were used in this search: ('fruit' OR 'vegetable' OR 'diet' OR 'dietary' OR 'nutrition') AND ('mental disorder' OR 'depression' OR 'anxiety' OR 'depressive disorder' OR ' mood disorder'). No time and language restrictions were applied. In addition, we reviewed the reference lists of the relevant publications and reviews to avoid missing any published study. Unpublished studies were not included in the search strategy.

\section{Inclusion criteria}

The studies included in our systematic review and meta-analysis were independently assessed by three investigators (F. S., P. S. and H. M.) separately, and publications that fulfilled the following criteria were eligible for inclusion in the present systematic review - population: adults; intervention/exposure: fruit or vegetable intakes; comparison: amount of consumption; outcome: depression or anxiety; study design: cross-sectional, case-control or cohort studies (case-control studies were not included in the meta-analysis).

\section{Exclusion criteria}

A total of 9557 articles were found in our initial search. We excluded letters, comments and animal studies from the analysis. Studies were excluded if they (1) had reported only the amount of fruit and/or vegetable intake across groups with and without mental disorders and did not provide any estimates for the association or did not provide any measures enabling us to calculate the effect size for the association ${ }^{(31-33)}$; (2) had examined major dietary patterns (including patterns highly loaded with fruit and vegetables) rather than fruit and/or vegetable intake separately ( $n$ 40); and (3) had examined the relation between fruit and vegetable intake and depression and/or anxiety in pregnant women $^{(34,35)}$ and children or adolescents ${ }^{(12,15,36-39)}$. We also excluded duplicate studies. When we found more than one published report based on the same study population ${ }^{(20)}$, only the most comprehensive publication ${ }^{(17)}$ was included in this meta-analysis. In addition, for one study that reported the association in several waves of a survey in Canada, we used data from the first wave only because data from some states were repeatedly used in other waves of the survey ${ }^{(5)}$. For another study $^{(40)}$ that reported the estimates for both predominant and completely vegetarians $v$. non-vegetarians, we used data from completely vegetarians (and not those from predominant vegetarians). After these exclusions, twenty-seven papers remained for systematic review in the present study (Fig. 1).

\section{Data extraction}

From each eligible study, we extracted the following information: first author, year of publication, study design, name of study, country, age range or mean age, sex, sample size, number of cases, exposure, exposure assessment tool, outcome, outcome assessment tool, relevant effect sizes (OR, hazard ratios (HR), RR, regression coefficients or Pearson's correlation coefficients) and 95\% CI and covariates that were adjusted for.

\section{Quality assessment of studies}

The quality of included studies was examined by using the Newcastle-Ottawa Scale (NOS) ${ }^{(41)}$. For cohort and case-control 


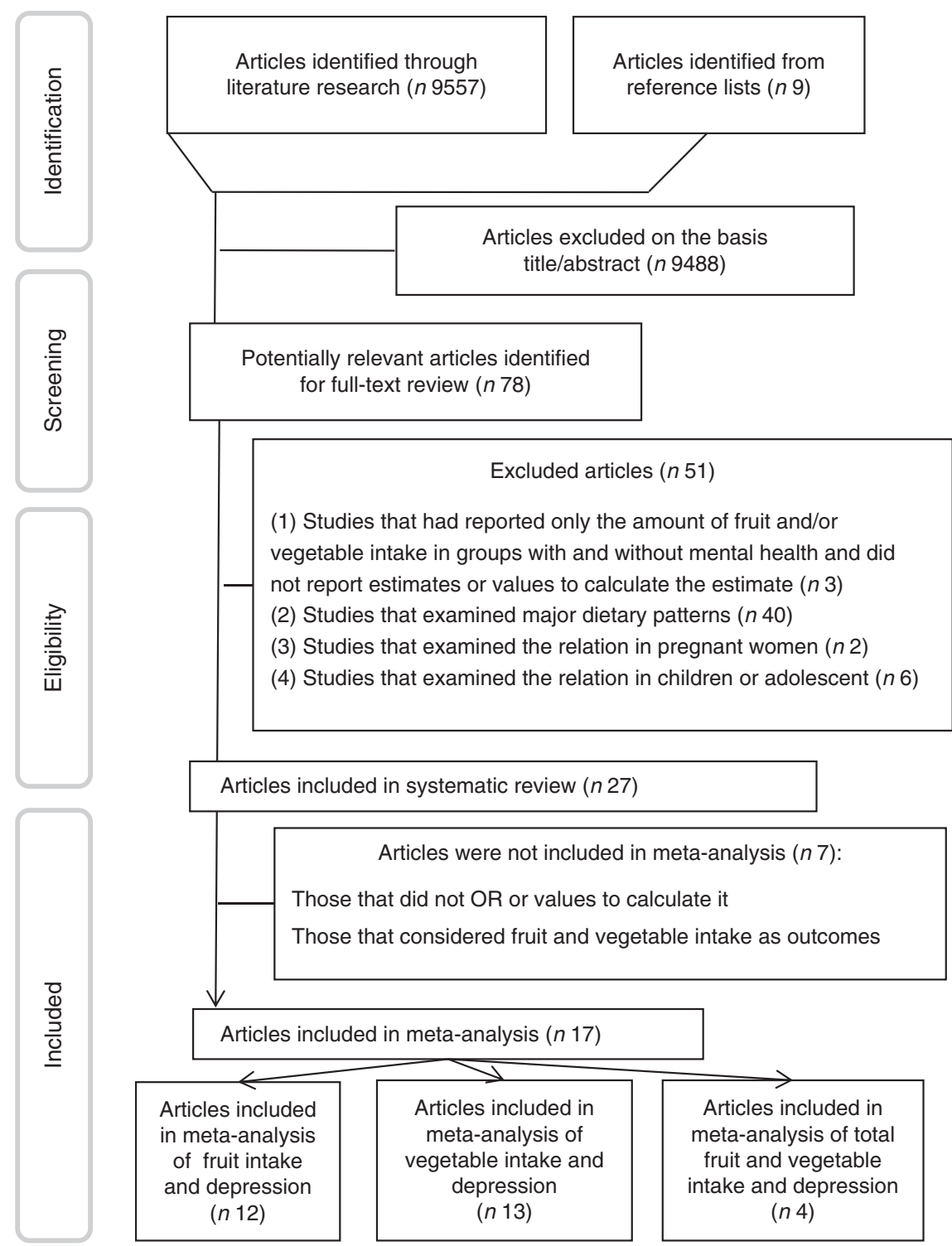

Fig. 1. The flow diagram of study selection.

studies that were included in the analysis, we used their own specific methods. For cross-sectional studies that were included in the analysis, we used the method for case-control studies, by considering subjects without depression or anxiety as the controls. The NOS assigns a maximum of nine points to each study: four for selection, two for comparability and three for assessment of outcomes (for cohort study) or exposures (for casecontrol study). The quality score ranged from 2 to 7 in this study, with the median of 5 . In the present analysis, when a study got more than median stars ( $\geq 5)$, it was considered to be of relatively high quality; otherwise, it was deemed to have low quality. Any discrepancies were resolved by discussion.

\section{Statistical analysis}

Reported RR, HR or OR (and their 95\% CI) were used to calculate $\log \mathrm{RR}$ and its standard errors. For five studies $^{(23,26,28,42,43)}$ that reported RR for the lowest $v$. the highest intake of fruit or vegetables, we inverted RR and its lower and upper limits to compute the RR for the highest $v$. the lowest intakes. For two studies ${ }^{(16,25)}$ that reported several RR for different kinds of fruit and vegetables, first we consolidated them in a preliminary meta-analysis using fixed-effects model and reached to a pooled RR for that study. Then, using a randomeffects model that takes between-study variation into account, the overall effect size from all included studies was calculated. Heterogeneity was assessed using Cochran's $Q$ test and $I^{2}$. In case of significant heterogeneity, we used subgroup analysis to explore possible sources of heterogeneity. Heterogeneity was examined through the random-effects model. Sensitivity analysis was performed to examine the extent to which inferences might depend on a particular study. Publication bias was assessed by visual inspection of Begg's funnel plots. Formal statistical assessment of funnel plot asymmetry was performed by Egger's regression asymmetry test. We also performed random-effects meta-regression analysis to assess the overall 
linear relationship between fruit or vegetable intake and risk of depression. In this analysis, RR (95\% CI) for depression in different categories of fruit or vegetable intake, compared with the reference group, were extracted. Next, they were converted to LnRR and were used in this meta-regression. We used a previously described method by Greenland and Orsini for the dose-response analysis ${ }^{(44)}$. The natural logs of RR and CI across categories of fruit or vegetable intake were used to compute study-specific slopes (linear trends) and 95\% CI. We assigned the median or mean amount of fruit or vegetable intake in each category to the corresponding RR for each study. For studies that reported the intakes as ranges, we estimated the mid-point in each category by calculating the mean of the lower and upper bound. When the highest category was open-ended, the length of the open-ended interval was assumed to be the same as that of the adjacent interval. When the lowest category was open-ended, the lower boundary was set to zero. We used $75 \mathrm{~g}$ as a vegetable serving and $150 \mathrm{~g}$ as a fruit serving. Restricted cubic spline (3) knots at fixed percentiles of 10, 50 and $90 \%$ of the distribution were considered to examine potential nonlinear dose-response associations between fruit or vegetable intake and risk of depression. Statistical analyses were conducted using STATA version 11.2 (StataCorp). $P$ values $<0 \cdot 05$ were considered statistically significant.

\section{Results}

\section{Findings from systematic review}

The characteristics of twenty-seven studies included in this systematic review are presented in Tables 1 and 2 . These studies were published between 2001 and 2017. Among included studies, sixteen had a cross-sectional design ${ }^{(5,14,16,21,23,25-28,43,45-50)}$, nine studies had a cohort design ${ }^{(10,17-19,22,29,30,42,51)}$ and the remaining two studies were case-control studies ${ }^{(40,52)}$. In all, ten publications were reported from European countries $^{(10,14,16,18,23,26,40,46,50,51)}$, five studies were reported from American countries $(5,22,45,49,52)$ and eight studies were reported from $\operatorname{Asia}^{(17,21,25,27,28,30,42,43)}$, and the remaining four studies were reported from Australia ${ }^{(19,29,47,48)}$. All published studies were conducted in adult populations. Four studies were conducted on women ${ }^{(19,22,45)}$, one on men only ${ }^{(49)}$ and twenty-two studies on both genders. Sample sizes ranged from seventy-one people in cross-sectional studies to 125428 in cohort studies. In total, 289018 participants were studied. The number of cases varied from 51 to 9739. Most included studies had used FFQ to assess dietary intakes, except for ten studies that had applied $24-\mathrm{h}$ recalls $^{(43,45)}$, dietary history ${ }^{(25,48)}$ and food questionnaire $e^{(21,23,27,28,49,51)}$. A total of sixteen studies had considered fruit intake $\mathrm{e}^{(5,10,16-19,21-23,28,29,42,46,48,50,52)}$, nineteen studies had assessed consumption of vegetables $^{(5,10,16-19,21,22,25,28-30,40,43,46-48,50,52)}$ and in seven other studies total consumption of fruit and vegetables was examined $^{(5,14,26,27,45,49,51)}$. One study had considered plant foods including vegetables, legumes, fruits and nuts as the exposure variable $^{47)}$. For assessment of mental disorders, nine studies used Center for Epidemiological Studies-Depression Scale $(\text { CES-D })^{(10,14,17,19,26,29,42,45,47,49)}$, five studies used Geriatric
Depression Scale ${ }^{(21,25,27,30,43)}$, four others had applied Beck's Depression Inventory $(\mathrm{BDI})^{(16,23,46,50,53)}$ and the remaining eight studies had used other questionnaires ${ }^{(5,18,28,40,48,51,52)}$.

With regard to outcome, only four studies had considered anxiety $^{(5,40,47,48)}$. One study had reported $\mathrm{OR}^{(40)}$, one had reported correlation coefficients ${ }^{(48)}$ and two studies had reported $\beta$-coefficients ${ }^{(5,47)}$. Owing to the heterogenous form of reporting of the findings, we were unable to perform metaanalysis about anxiety. All studies had considered depression. Four studies reported beta coefficients ${ }^{(46,47,49-52)}$, one had reported standardised $\beta^{(45)}$, one reported correlation coefficient $^{(48)}$ and others had reported RR, HR or OR. OR for the association of fruit intake and depression ranged between 0.61 and 1.10 in different studies. The corresponding figures for vegetable intake varied from 0.63 to 2.75 . A total of fourteen studies were of high quality ${ }^{(5,10,14,17-19,25,27-30,47,50,51)}$. Most studies had adjusted for age ${ }^{(5,10,14,16,18,21,25-28,30,40,43,45-47,52)}$, $\operatorname{sex}^{(5,16,18,21,25,26,28,42,43,46,47,50,52)}$, education $(5,14,16,21,22,25,28,29$,' $40,42,45,47,49,52)$, physical activity ${ }^{(5,10,14,16,18,22,25,26,29,47)}$, energy intake $^{(10,14,18,25,26,29,45,47)}, \quad \operatorname{BMI}^{(14,18,22,25,26,29,47,52)}$ and smoking ${ }^{(5,10,16-18,22,25,28,29,42,47)}$. Some had also controlled for income $^{(5,10,21,22,26,29,49)}$, marital status ${ }^{(10,16,18,25,28,29,40,45)}$ and chronic diseases ${ }^{(5,10,17,21,22,25,27)}$.

Cross-sectional design ${ }^{(5,14,16,21,23,25,27,42,45-47,50,52)}$, invalid exposure or outcome assessment tools ${ }^{(5,10,17,19,21,25,42,43,49-51)}$, lack of controlling for potential confounders $(16,18,22,25,30,45,51)$, using self-reported questionnaire ${ }^{(14,17,18,28,30,50,52)}$, low respondent rate ${ }^{(19,30,51)}$ and misclassification of participants based on assessing dietary intake by $\mathrm{FFQ}^{(18,22)}$ were the most common limitations of earlier studies. However, large sample size $\mathrm{e}^{(5,14,19,21,29,42,51)}$, adjustment for most potential confounders ${ }^{(5,14,29)}$, valid exposure or outcome assessment tools $(19,21,29,42,45,51)$ and the first study in a special population ${ }^{(28,45)}$ were mostly reported as strengths of these publications.

\section{Findings from the meta-analysis of fruit intake and risk of depression}

Combining six effect sizes from six cohort studies ${ }^{(10,17-19,22,29)}$, we found that the highest $v$. the lowest intake of fruit was associated with a $17 \%$ significant reduction in the risk of depression (Fig. 2) (overall $\mathrm{RR}=0.83 ; 95 \%$ CI 0.71 , 0.98). However, heterogeneity was significant $\left(I^{2}=84.5 \%, P<0 \cdot 001\right)$. To investigate the source of heterogeneity, subgroup analyses were performed on the basis of location, sex, outcome assessment tools and study quality (Table 3 ). Sex (for female: overall $\mathrm{RR}=0.92 ; 95 \%$ CI $0.78,0.98, I^{2}=87.3 \%, P<0.001$, and for both: overall RR $=0.72 ; 95 \%$ CI $\left.0.61,0.84, I^{2}=7 \%, P=0.34\right)$ and location (for Asian countries: overall $\mathrm{RR}=0 \cdot 87$; $95 \% \mathrm{CI} 0.78$, $0.96, I^{2}$ squared $=0 \%, P=0.62$, and for non-Asian countries: overall RR $=0.81 ; 95 \%$ CI 0.62, $1 \cdot 05, I^{2}=89 \cdot 1 \%, P<0 \cdot 001$ ) were the sources of heterogeneity. Combining nine effect sizes from six cross-sectional studies ${ }^{(5,16,21,23,24,28)}$ indicated that the highest $v$. the lowest intake of fruit was associated with a $24 \%$ reduction in the risk of depression (overall $\mathrm{RR}=0.76$; $95 \% \mathrm{CI}$ $0.63,0.92, I^{2}=82.7 \%, P<0.001$ ) (Fig. 3). To investigate the source of heterogeneity, subgroup analysis was done on the basis of location, sex, dietary assessment tools, outcome 
Table 1. Characteristics of studies that reported the relationship between fruit and vegetable intake and depression

(Odds ratios, relative risks (RR), hazard ratios (HR) and $95 \%$ confidence intervals; mean values and $\beta$-coefficients with their standard errors)

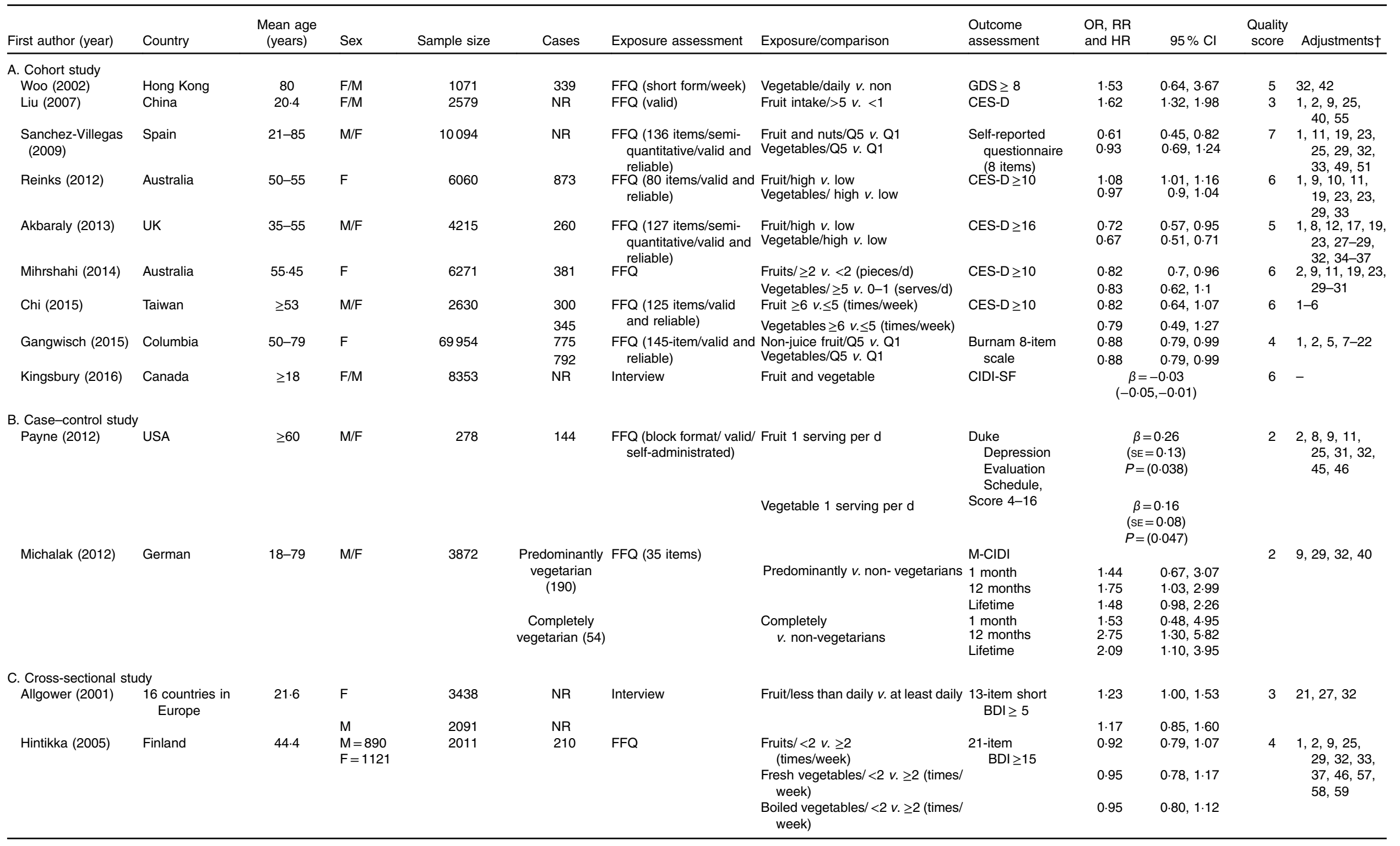


Table 1. Continued

\begin{tabular}{|c|c|c|c|c|c|c|c|c|c|c|c|c|}
\hline First author (year) & Country & $\begin{array}{l}\text { Mean age } \\
\text { (years) }\end{array}$ & Sex & Sample size & Cases & Exposure assessment & Exposure/comparison & $\begin{array}{l}\text { Outcome } \\
\text { assessment }\end{array}$ & $\begin{array}{l}\text { OR, RR } \\
\text { and HR }\end{array}$ & $95 \% \mathrm{Cl}$ & $\begin{array}{l}\text { Quality } \\
\text { score }\end{array}$ & Adjustments $\dagger$ \\
\hline \multirow[t]{2}{*}{ Woo (2006) } & Hong Kong & $\geq 65$ & $\mathrm{M} / \mathrm{F}$ & 3994 & 280 & 7-d FFQ & $\begin{array}{l}\text { Fruits and dried fruits } / 2334 \mathrm{~g} / \\
\text { week } \leq v . \leq 1077 \mathrm{~g} / \mathrm{week}\end{array}$ & $\mathrm{GDS} \geq 8$ & 0.71 & $0.48,1.05$ & 2 & $\begin{array}{c}5,9,14,17,25 \\
32,37,56\end{array}$ \\
\hline & & & & & & & $\begin{array}{l}\text { Vegetables } / 2112 \mathrm{~g} / \text { week } \leq v \\
1036 \geq \mathrm{g} / \text { week }\end{array}$ & & 0.63 & $0.44,0.92$ & & \\
\hline \multirow[t]{5}{*}{ Mikolajczyk (2009) } & $\begin{array}{l}\text { Germany } \\
\text { Poland }\end{array}$ & $\begin{array}{l}20 \cdot 6 \\
\text { (SE 2.3) }\end{array}$ & $F=1244$ & $\begin{array}{c}\text { Germany ( } n \text { 696), } \\
\text { Poland ( } n \text { 489) }\end{array}$ & NR & $\begin{array}{l}\text { FFQ (12 indicator } \\
\text { variable/ valid) }\end{array}$ & Fruits & M-BDI & \multicolumn{2}{|c|}{$\begin{array}{l}{ }^{*} \beta=-1.69 \\
(P=0.002)\end{array}$} & 5 & $25,27,28$ \\
\hline & Bulgaria & & & Bulgaria ( $n$ 654) & & & Salads & & \multicolumn{2}{|c|}{$\begin{array}{l}{ }^{*} \beta=-2.55 \\
(P<0.001)\end{array}$} & & \\
\hline & & & $M=645$ & & & & Fruit & & \multicolumn{2}{|c|}{$\begin{array}{c}{ }^{*} \beta=-0.45 \\
(P=0.53)\end{array}$} & & \\
\hline & & & & & & & Salads & & \multicolumn{2}{|c|}{$\begin{array}{l}{ }^{*} \beta=-0.88 \\
(P=0.25)\end{array}$} & & \\
\hline & & & & & & & Vegetable & & \multicolumn{2}{|c|}{$\begin{array}{l}{ }^{*} \beta=0.77 \\
(P=0.29)\end{array}$} & & \\
\hline Verger (2009) & France & $\geq 18$ & $\mathrm{M} / \mathrm{F}$ & 10279 & NR & $\mathrm{FFQ}$ & $\begin{array}{l}\text { Fruit and vegetable/non-daily } v \text {. } \\
\text { daily }\end{array}$ & $\begin{array}{l}\text { CES-D }<17 \text { in } \\
\text { men and } \\
\text { CES-D }<23 \text { in } \\
\text { women }\end{array}$ & 1.49 & $1 \cdot 26,1 \cdot 76$ & 4 & $\begin{array}{l}10,11,25,32 \\
52-54\end{array}$ \\
\hline \multirow[t]{2}{*}{ Aihara (2011) } & Japan & $76 \cdot 1$ & M & 416 & 51 & \multirow{2}{*}{$\begin{array}{l}\text { Questionnaire based on } \\
\text { MAFF-2000 }\end{array}$} & \multirow{2}{*}{$\begin{array}{l}\text { Vegetable and fruit/enough } v \text {. } \\
\text { not enough }\end{array}$} & $\mathrm{GDS} \geq 2$ & 1.52 & $0.74,3 \cdot 10$ & 6 & \multirow[t]{2}{*}{$6,32,38$} \\
\hline & & 74.9 & $\mathrm{~F}$ & 471 & 63 & & & (5-item) & 1.53 & $0.84,2.77$ & & \\
\hline Castellanos (2011) & USA & $\begin{array}{c}29 \cdot 6 \\
(\mathrm{SE} 8 \cdot 2)\end{array}$ & M & 75 & NR & $\begin{array}{l}\text { The Block fat and fruit } \\
\text { and vegetable } \\
\text { screening tool for } \\
\text { Mexican Americans }\end{array}$ & Fruit and vegetable & CES-D $\geq 16$ & & $\begin{array}{l}=0.30 \\
<0.05)\end{array}$ & 2 & $9,10,32,44$ \\
\hline Shahar (2011) & Malaysia & $68.56-70 \cdot 44$ & $\mathrm{M} / \mathrm{F}$ & 71 & 51 & $\begin{array}{l}\text { 1-d food weighing } \\
24-h \text { diet recall }\end{array}$ & Vegetables/low v. high & $\mathrm{GDS} \geq 5$ & 3.31 & $1.03,10.60$ & 2 & $8,25,32$ \\
\hline \multirow[t]{2}{*}{ Forsyth (2012) } & Australia & $>18$ & $\mathrm{M} / \mathrm{F}$ & 109 & NR & $\begin{array}{l}\text { Comprehensive diet } \\
\text { history }\end{array}$ & Fruit & DASS & \multicolumn{2}{|c|}{$\begin{array}{l}r-0.31 \\
P<0.01\end{array}$} & 1 & - \\
\hline & & & & & & & Vegetable & & \multicolumn{2}{|c|}{$\begin{array}{l}r-0.24 \\
P<0.05\end{array}$} & & \\
\hline Crichton (2013) & Australia & $40-65$ & $\mathrm{M} / \mathrm{F}$ & 1183 & 103 & $\begin{array}{l}\text { FFQ (215 items/valid } \\
\text { and reliable) }\end{array}$ & $\begin{array}{l}\text { Plant foods (vegetables, legumes, } \\
\text { fruit, nuts) }\end{array}$ & CES-D & $\beta=-0.237$ & $(-0.422,-0.52)$ & 5 & $\begin{array}{l}1,9,11,19,23 \\
32\end{array}$ \\
\hline \multirow[t]{4}{*}{ Niu (2013) } & Japan & $>70$ & $\mathrm{M} / \mathrm{F}$ & 986 & $\begin{array}{c}\text { Mild }=344 \\
\text { Sever }=199\end{array}$ & $\begin{array}{l}\text { Diet history } \\
\text { questionnaire }\end{array}$ & $\begin{array}{l}\text { Tomatoes and tomato products } \\
\text { Green-leaf vegetables }\end{array}$ & GDS & $\begin{array}{l}0.48 \\
0.72\end{array}$ & $\begin{array}{l}0.31,0.75 \\
0.45,1.15\end{array}$ & 6 & $1,5,6,9,11$ \\
\hline & & & & & & & Cabbage & with 2 cut-off & 1.46 & $0.85,2.50$ & & $23,25,29$ \\
\hline & & & & & & & $\begin{array}{l}\text { Carrot, onion, burdock, lotus root } \\
\text { and pumpkin }\end{array}$ & points & 1.34 & $0.74,2.45$ & & $32,41-45$ \\
\hline & & & & & & & $\begin{array}{l}\text { Japanese white radish (daikon) } \\
\text { and turnips }\end{array}$ & & 0.70 & $0.43,1.13$ & & \\
\hline
\end{tabular}




\section{NS British Journal of Nutrition}

Table 1. Continued

\begin{tabular}{|c|c|c|c|c|c|c|c|c|c|c|c|c|}
\hline First author (year) & Country & $\begin{array}{l}\text { Mean age } \\
\text { (years) }\end{array}$ & Sex & Sample size & Cases & Exposure assessment & Exposure/comparison & $\begin{array}{l}\text { Outcome } \\
\text { assessment }\end{array}$ & $\begin{array}{l}\mathrm{OR}, \mathrm{RR} \\
\text { and } \mathrm{HR}\end{array}$ & $95 \% \mathrm{Cl}$ & $\begin{array}{l}\text { Quality } \\
\text { score }\end{array}$ & Adjustments $\dagger$ \\
\hline \multirow[t]{2}{*}{ McMartin (2013) } & Canada & $\geq 12$ & $\mathrm{M} / \mathrm{F}$ & 125428 & 9739 & FFQ & $\begin{array}{l}\text { Fruit and vegetable/Q4 v. Q1 } \\
\text { Fruit/Q4 v. Q1 }\end{array}$ & CIDI-SF $\geq 5$ & $\begin{array}{l}0.85 \\
0.97\end{array}$ & $\begin{array}{l}0.78,0.92 \\
0.87,1.08\end{array}$ & 5 & $\begin{array}{c}1,9,10,19,25 \\
32,38\end{array}$ \\
\hline & & & & & & & Vegetable/Q4 v. Q1 & & $\begin{array}{l}0.91 \\
* R=-\end{array}$ & $0.83,1.01$ & & \\
\hline Whitaker (2014) & Columbia & $25-51$ & $\mathrm{~F}$ & 196 & NR & $\begin{array}{l}\text { Three 24-h dietary } \\
\text { recalls }\end{array}$ & Fruit and vegetables (serve/d) & $\begin{array}{l}\text { Short form of } \\
\text { CES-D }\end{array}$ & \multicolumn{2}{|c|}{${ }^{\star} B=-1.06(0.29)$} & 4 & $\begin{array}{c}9,23,29 \\
32,33\end{array}$ \\
\hline \multirow[t]{4}{*}{ Ansari (2014) } & UK & $\begin{array}{c}24.9 \\
(\mathrm{SE} 8 \cdot 6)\end{array}$ & $\mathrm{M} / \mathrm{F}$ & $M=761$ & NR & FFQ (12 indicator/ valid) & Fresh fruits & M-BDI & \multirow{2}{*}{\multicolumn{2}{|c|}{$\begin{array}{l}\beta=-0.074 \\
(P=0.047) \\
\beta=-0.091 \\
(P=0.014)\end{array}$}} & 3 & 25,26 \\
\hline & & & & & & & Salad/raw vegetables & & & & & \\
\hline & & & & $F=2706$ & NR & & Fresh fruits & & \multicolumn{2}{|c|}{$\begin{array}{c}\beta=-0.111 \\
(P<0.001)\end{array}$} & & \\
\hline & & & & & & & Salad/raw vegetables & & \multicolumn{2}{|c|}{$\begin{array}{c}\beta=-0.071 \\
(P<0.001)\end{array}$} & & \\
\hline \multirow[t]{6}{*}{ Bishwajit (2017) } & Bangladesh & \multirow[t]{6}{*}{$\geq 18$} & \multirow[t]{6}{*}{$\mathrm{F} / \mathrm{M}$} & 3262 & 1043 & Interview & Vegetable/ $>5 \quad v .<5($ serve $/ d)$ & \multirow{6}{*}{$\begin{array}{l}\text { Self-reported } \\
\text { questionnaire }\end{array}$} & 0.67 & $0.44,1.03$ & \multirow[t]{6}{*}{6} & \multirow{6}{*}{$\begin{array}{c}1,2,9,25,29 \\
32,33\end{array}$} \\
\hline & & & & & & & Fruit/>5 v. $<5$ (serve/d) & & 0.81 & $0.51,1.30$ & & \\
\hline & India & & & 7594 & 1344 & & Vegetable/ $>5$ v. $<5($ serve $/ d)$ & & 1.08 & $0.73,1.90$ & & \\
\hline & & & & & & & Fruit $/>5$ v. $<5$ (serve/d) & & $1 \cdot 10$ & $0.74,1.65$ & & \\
\hline & Nepal & & & 3277 & 1635 & & Vegetable/ $>5$ v. $<5($ serve $/ d)$ & & 0.99 & $0.57,1.72$ & & \\
\hline & & & & & & & Fruit $/>5 \quad$ v. $<5$ (serve/d) & & 1.06 & $0.79,1.42$ & & \\
\hline
\end{tabular}

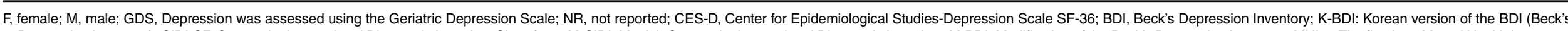

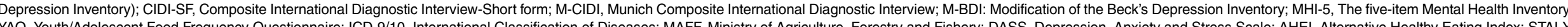

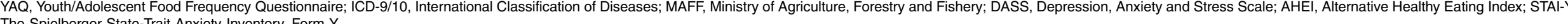
The Spielberger State-Trait Anxiety Inventory, Form $Y$.

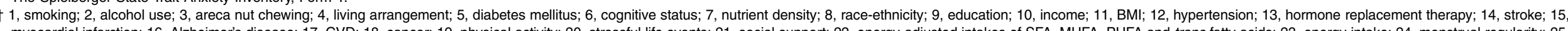

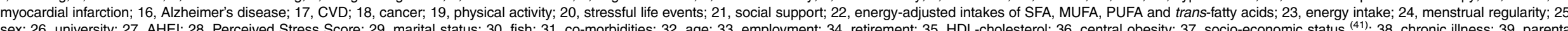

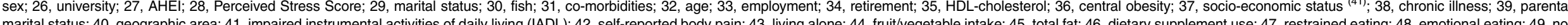

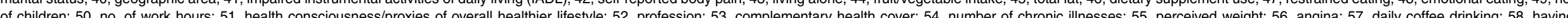

vocational training: 59, poor subjective health; 60, frequency of eating lake fish, sea fish, fresh vegetables, boiled vegetables and fruit. 
assessment tools, energy adjustment and study quality (Table 3). Location (for Asian countries: overall RR $=0.62 ; 95 \%$ CI $0.41,0.95, I^{2}=88.6 \%, P<0.001$, and for non-Asian countries: overall $\left.\mathrm{RR}=0.93 ; 95 \% \mathrm{CI} 0.86,1.01, I^{2}=0 \%, P=0.62\right)$ and outcome assessment tools (for BDI: overall RR $=0.89 ; 95 \% \mathrm{CI}$ $0.78,1.01, I^{2}=0 \%, P=0.72$ ), for self-reported questionnaire: overall RR $=0.58 ; 95 \% \mathrm{CI} 0.24,1.38, I^{2}=93.2 \%, P<0.001$, and for other outcome assessment tools: overall RR $=0.76 ; 95 \% \mathrm{CI}$ $\left.0.54, \quad 1.07, \quad I^{2}=87.5 \%, \quad P<0.001\right)$ were the sources of heterogeneity.

For non-linear dose-response meta-analysis on fruit consumption, there was only one cohort study that had provided relevant information. Therefore, we excluded this cohort study from the dose-response analysis owing to insufficient number of cohort studies. Finally, the non-linear dose-response analysis on fruit consumption was confined to two cross-sectional studies $^{(21,28)}$, which had provided four effect sizes. In this nonlinear dose-response analysis, we found that increased intake of fruit was not associated with reduced odds of depression $\left(P_{\text {non-linearity }}=0 \cdot 12\right)$ (Fig. 4).

On the basis of meta-regression on three effect sizes from three cohort studies ${ }^{(17,19,22)}$, we found an inverse linear association between fruit intake and risk of depression, such that every 100-g increase in intake of fruit was associated with a $3 \%$ reduction in the risk of depression ( $\mathrm{RR}=0.97 ; 95 \% \mathrm{CI} 0.95$, $0.99)$. With regard to cross-sectional studies ${ }^{(16,21,28)}$, fruit intake was not linearly associated with the risk of depression $(\mathrm{RR}=$ $1.00 ; 95 \%$ CI $0.99,1.01)$. Sensitivity analysis showed that the overall effect did not vary substantially with the exclusion of any study. The Begg's test $(P=0.46)$ and Egger's test $(P=0.07)$ had shown no publication bias.

\section{Findings from the meta-analysis of vegetable intake and risk of depression}

Summary effect from seven effect sizes provided by seven cohort studies $^{(10,17-19,22,29,30)}$ showed that high intake of vegetables was associated with a $14 \%$ significant reduction in the risk of depression (overall $\mathrm{RR}=0.86$; $95 \%$ CI $0.75,0.98$, $I^{2}=66.1 \%, P=0.004$ ) (Fig. 5). To find the source of heterogeneity, subgroup analysis was conducted on the basis of location, sex, outcome assessment tools and study quality. Sex (for female: overall $\mathrm{RR}=0.93 ; 95 \%$ CI $0.86,1 \cdot 00, I^{2}=27 \cdot 4$, $P=0.25$, and for both: overall $\mathrm{RR}=0.81 ; 95 \%$ CI $0.64,1.02$, $I^{2}=53.8 \%, P=0.09$ ), location (Asian countries: overall $\mathrm{RR}=$ 0.88 ; $95 \%$ CI $0.79,0.98, I^{2}=0 \%, P=0.38$, and for non-Asian countries: overall $\mathrm{RR}=0.84 ; 95 \%$ CI $0.68,1.04, I^{2}=81.9 \%$, $P=0.001$ ) and outcome assessment tools (for CES-D: overall $\mathrm{RR}=0.81 ; 95 \% \mathrm{CI} 0.65,1.02, I^{2}=82.5 \%, P=0.001$, and for other outcome assessment tools: overall $\mathrm{RR}=0.89 ; 95 \% \mathrm{CI} 0 \cdot 80,0 \cdot 99$, $I^{2}=0 \%, P=0.45$ ) were the sources of heterogeneity (Table 4 ). Combining eight effect sizes from six cross-sectional studies $^{(5,16,21,25,28,43)}$ indicated that higher intake of vegetables was associated with a $25 \%$ reduced risk of depression compared with lower intake of vegetables (overall $\mathrm{RR}=0.75$; $95 \%$ CI $0.62,0.91, I^{2}=56.8 \%, P=0.023$ ) (Fig. 6). Subgroup analysis on the basis of location, dietary assessment tools, outcome assessment tools, energy adjustment and quality score 


\begin{tabular}{|c|c|c|c|}
\hline First author (year) & & $\mathrm{RR} 95 \% \mathrm{Cl}$ & Weight (\%) \\
\hline Sanchez (2009) & & $0.610 .45,0.82$ & $12 \cdot 53$ \\
\hline Akbaraly (2013) & & $0.720 .57,0.95$ & $14 \cdot 21$ \\
\hline Reinkes (2013) & $\rightarrow$ & $1 \cdot 081 \cdot 01,1 \cdot 16$ & $21 \cdot 11$ \\
\hline Mihrshahi (2014) & & $0.820 .70,0.96$ & $18 \cdot 17$ \\
\hline Gangwisch (2015) & & $0.880 .79,0.99$ & $19 \cdot 84$ \\
\hline Chi (2015) & - & $0.820 .64,1.07$ & $14 \cdot 15$ \\
\hline Overall $\left(I^{2}=84.5 \%, P=0.000\right)$ & & $0.830 .71,0.98$ & $100 \cdot 00$ \\
\hline 0.45 & 1 & $2 \cdot 22$ & \\
\hline
\end{tabular}

Fig. 2. Forest plots of the association between fruit consumption and risk of depression in cohort studies. RR, relative risk.

Table 3. Results of subgroup analysis for fruit intake and risk of depression based on study design (Odds ratios and $95 \%$ confidence intervals)

\begin{tabular}{|c|c|c|c|c|c|c|}
\hline & No. of effect sizes & Ref. & OR & $95 \% \mathrm{Cl}$ & $I^{2}(\%)$ & $P_{\text {Heterogeneity }}$ \\
\hline Cohort studies & 6 & & 0.83 & $0.71,0.98$ & 84.5 & $>0.001$ \\
\hline \multicolumn{7}{|l|}{ Asian $v$. non-Asian } \\
\hline Asian & 2 & $(17,22)$ & 0.87 & $0.78,0.96$ & 0 & 0.62 \\
\hline Non-Asian & 4 & $(10,18,19,29)$ & 0.81 & $0.63,1.05$ & $89 \cdot 1$ & $>0.001$ \\
\hline \multicolumn{7}{|l|}{ Sex } \\
\hline Female & 3 & $(19,22,29)$ & 0.93 & $0.78,1.11$ & $87 \cdot 3$ & $>0.001$ \\
\hline Both & 3 & $(17,18,54)$ & 0.72 & $0.61,0.85$ & $7 \cdot 2$ & 0.34 \\
\hline \multicolumn{7}{|l|}{ Outcome assessment tools } \\
\hline CES-D & 4 & $(10,17,19,29)$ & 0.87 & $0.70,1.07$ & 84.6 & $>0.001$ \\
\hline Other tools & 2 & $(18,22)$ & 0.75 & $0.53,1.07$ & 80.1 & 0.025 \\
\hline \multicolumn{7}{|l|}{ Study quality } \\
\hline High quality (score $\geq 5$ ) & 5 & $(17-19,29,54)$ & 0.81 & $0.65,1.01$ & $86 \cdot 4$ & $>0.001$ \\
\hline Low quality (score $<5$ ) & 1 & (22) & 0.88 & $0.79,0.98$ & - & - \\
\hline Cross-sectional study & 9 & $(5,16,21,23,28,42)$ & 0.76 & $0.63,0.92$ & $82 \cdot 7$ & $>0.001$ \\
\hline \multicolumn{7}{|l|}{ Asian v. non-Asian } \\
\hline Asian & 5 & $(21,28,42)$ & 0.62 & $0.41,0.95$ & 88.6 & $>0.001$ \\
\hline Non-Asian & 4 & $(5,16,23)$ & 0.93 & $0.86,1.01$ & 0 & 0.626 \\
\hline \multicolumn{7}{|l|}{ Sex } \\
\hline Male & 1 & (23) & 0.85 & $0.62,1.17$ & - & - \\
\hline Female & 1 & (23) & 0.81 & $0.65,1.17$ & - & - \\
\hline Both & 7 & $(5,16,21,28,42)$ & 0.74 & $0.58,0.93$ & 86.9 & $>0.001$ \\
\hline \multicolumn{7}{|l|}{ Dietary assessment tools } \\
\hline $\mathrm{FFQ}$ & 4 & $(5,16,21,42)$ & 0.81 & $0.66,1.00$ & $81 \cdot 8$ & 0.001 \\
\hline Other tools & 5 & $(23,28)$ & 0.69 & $0.46,1.03$ & 86.4 & $>0.001$ \\
\hline \multicolumn{7}{|l|}{ Outcome assessment tools } \\
\hline $\mathrm{BDI}$ & 3 & $(16,23)$ & 0.89 & $0.78,1.01$ & 0 & 0.72 \\
\hline Self-reported questionnaire & 3 & (28) & 0.58 & $0.24,1.38$ & $93 \cdot 2$ & $>0.001$ \\
\hline Other tools & 3 & $(5,21,42)$ & 0.76 & $0.54,1.07$ & 87.5 & $>0.001$ \\
\hline \multicolumn{7}{|l|}{ Energy adjusted } \\
\hline Adjusted & 4 & $(5,28)$ & 0.69 & $0.45,1.06$ & $90 \cdot 2$ & $>0.001$ \\
\hline Non-adjusted & 5 & $(16,21,23,42)$ & 0.78 & $0.65,0.93$ & $60 \cdot 1$ & 0.040 \\
\hline \multicolumn{7}{|l|}{ Study quality } \\
\hline High quality (score $\geq 5$ ) & 4 & $(5,28)$ & 0.69 & $0.45,1.06$ & $90 \cdot 2$ & $>0.001$ \\
\hline Low quality $($ score $<5)$ & 5 & $(16,21,23,42)$ & 0.78 & $0.65,0.93$ & 60.1 & 0.040 \\
\hline
\end{tabular}

CES-D, Center for Epidemiological Studies-Depression Scale SF-36; BDI, Beck's Depression Inventory.

were performed to investigate the source of heterogeneity (Table 4). None of these variables explained heterogeneity.

A non-linear dose-response meta-analysis of cohort studies on vegetable consumption ${ }^{(19,22,30)}$ revealed that increased intake of vegetables (at the level of 100-400 g/d) was associated with a reduced risk of depression; however, vegetable consumption in excess of $400 \mathrm{~g} / \mathrm{d}$ was associated with an increased risk of depression (Fig. 7(a)). However, these 


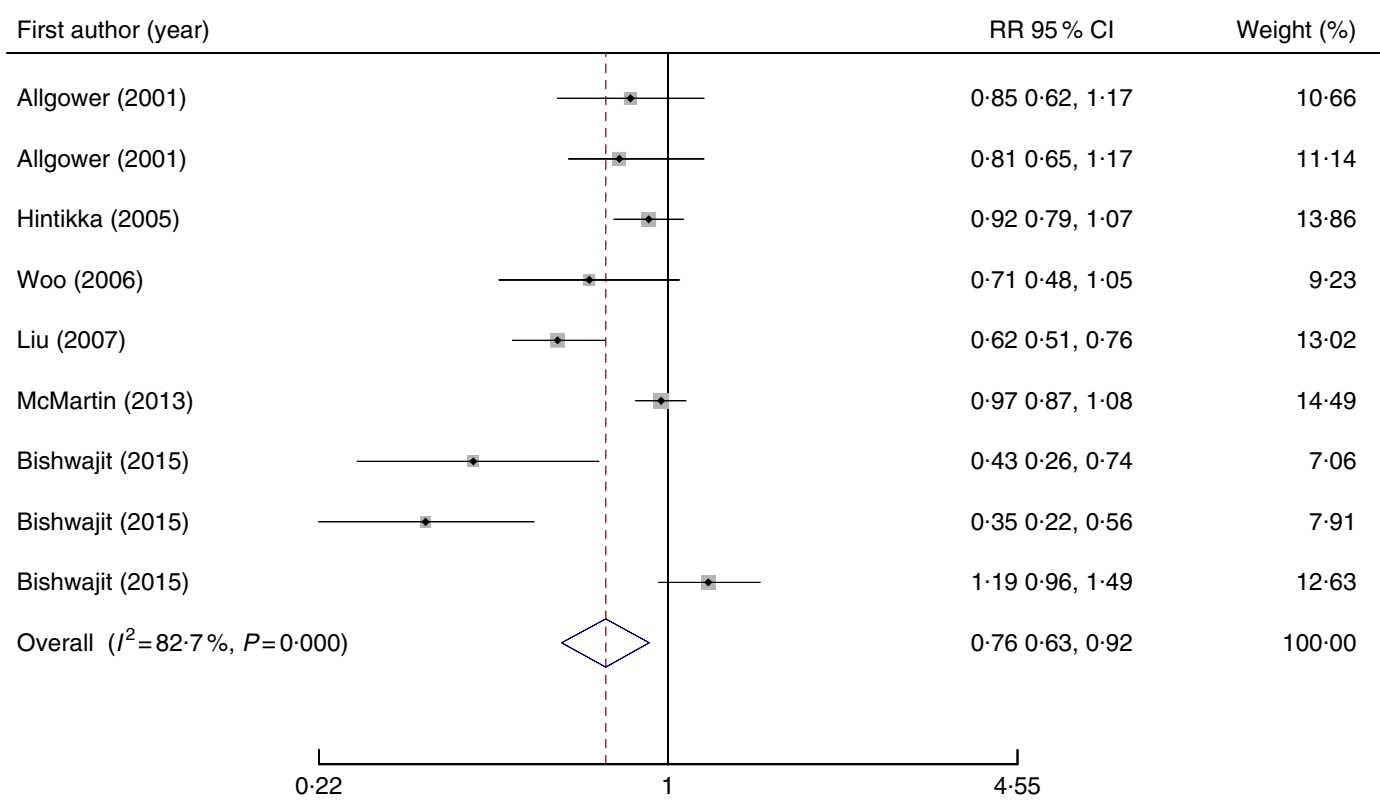

Fig. 3. Forest plots of the association between fruit consumption and depression in cross-sectional studies. RR, relative risk.

findings were not statistically significant $\left(P_{\text {non-linearty }}=0.97\right)$. The non-linear dose-response meta-analysis of cross-sectional studies $^{(21,25,28)}$ indicated that increased intake of vegetables was associated with a reduced risk of depression $\left(P_{\text {non-linearty }}<0 \cdot 001\right)$ (Fig. 7(b)).

We performed linear meta-regression analysis because of a non-significant non-linear association between vegetable consumption and risk of depression. Four effect sizes from four cohort studies ${ }^{(17,19,22,30)}$ were included in linear meta-regression. We found that every 100 -g increase in consumption of vegetables was associated with a $3 \%$ reduced risk of depression ( $\mathrm{RR}=0.97,95 \% \mathrm{CI} 0.95,0.98)$. With regard to cross-sectional studies, combining six effect sizes from four studies $^{(16,21,25,28)}$, we found that every 100 -g increased intake of vegetables was associated with a $5 \%$ reduced odds of depression ( $R R=0.95 ; 95 \%$ CI $0.91,0.98)$. Sensitivity analysis showed that none of the studies had a significant effect on the overall effect size. No evidence of publication bias was seen (Egger's test: $P=0 \cdot 20$, Begg's test: $P=0 \cdot 56$ ).

\section{Findings from the meta-analysis on total fruit and vegetable intake and risk of depression}

Overall effect from six effect sizes, obtained from four crosssectional studies ${ }^{(5,14,26,27)}$, revealed that high intake of fruit and vegetables was associated with a $20 \%$ reduced risk of depression (Fig. 8) (overall RR =0.80; 95\% CI 0.65, 0.98). However, significant heterogeneity was observed between studies $\left(I^{2}=71 \cdot 1 \%, P=0 \cdot 004\right)$. We performed subgroup analysis on the basis of sex, location, dietary assessment tools, outcome assessment tools and quality score to investigate the source of heterogeneity (Table 5). Location (for Asian countries: overall $\mathrm{RR}=1.53 ; 95 \%$ CI $0.96,2 \cdot 41, I^{2}=0 \%, P=0.99$, and for nonAsian countries: overall RR $=0.72 ; 95 \%$ CI 0.60, 0.87, $I^{2}=69 \cdot 1 \%$, $P=0.02$ ) and dietary assessment tools (for FFQ: overall

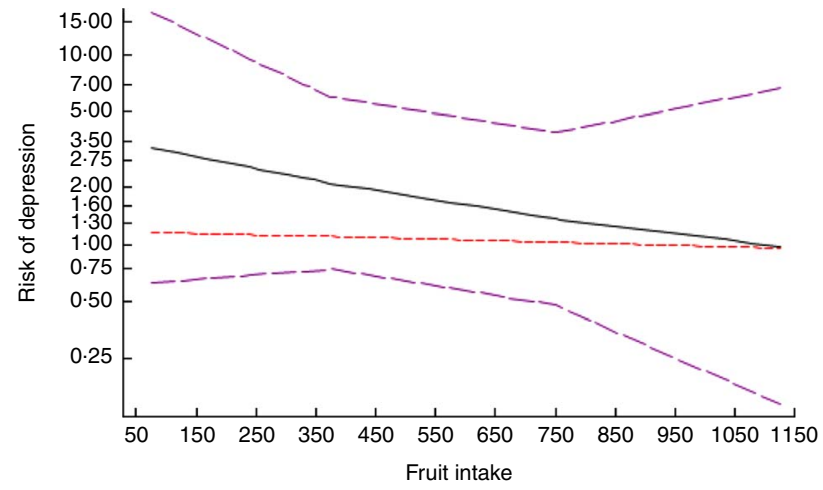

Fig. 4. Dose-response association between fruit consumption and risk of depression in cross-sectional studies. -----, Linear model; _spline model.

$\mathrm{RR}=0.72 ; 95 \%$ CI $0.60,0.87, I^{2}=69.1 \%, P=0.02$, and for questionnaire: overall $\mathrm{RR}=1.53$; $95 \%$ CI $0.96,2 \cdot 41, I^{2}=0 \%$, $P=0.99)$ were the sources of heterogeneity. Sensitivity analysis revealed that none of the studies had significantly influenced the overall effect. No evidence of publication bias was found (Egger's test: $P=0.82$, Begg's test: $P=0.57$ ).

\section{Discussion}

In this meta-analysis on eighteen studies, we found that high consumption of fruit, vegetables and total fruit and vegetables was significantly associated with reduced risk of depression. However, the magnitude of this association was dependent on the type of studies. We also found that every 100-g increase in the consumption of fruit or vegetables was associated with a $5 \%$ reduction in the risk of depression.

Depression is a serious and common mental disorder, which imposes a substantial burden to both individuals and societies. It is expected to be the first cause of disease and disability by 


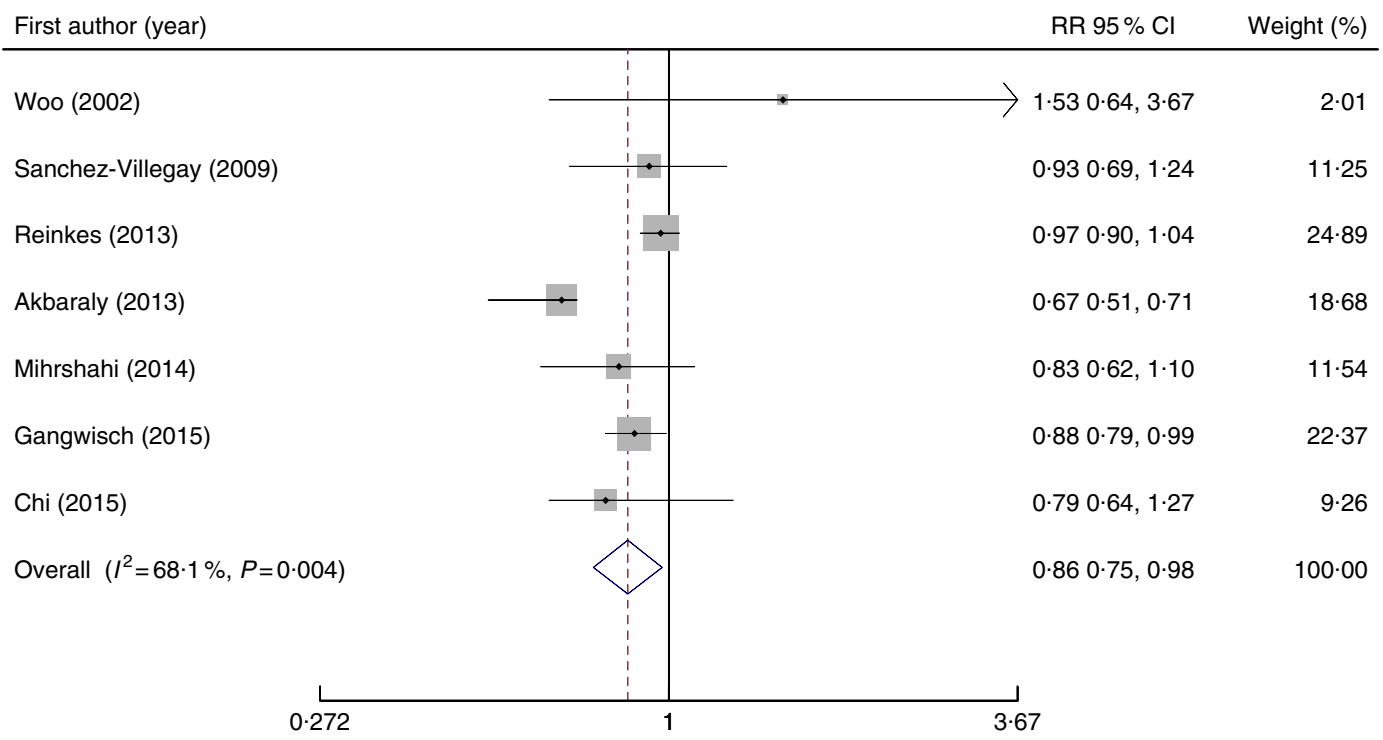

Fig. 5. Forest plots of the association between consumption of vegetables and risk of depression in cohort studies. RR, relative risk.

Table 4. Results of subgroup analysis for vegetable intake and risk of depression based on study design (Odds ratios and $95 \%$ confidence intervals)

\begin{tabular}{|c|c|c|c|c|c|c|}
\hline & No. of effect sizes & Ref. & OR & $95 \% \mathrm{Cl}$ & $P(\%)$ & $P_{\text {Heterogeneity }}$ \\
\hline Cohort study & 7 & $(10,17-19,22,29,30)$ & 0.86 & $0.75,0.97$ & $66 \cdot 1$ & 0.004 \\
\hline \multicolumn{7}{|l|}{ Asian $v$. non-Asian } \\
\hline Asian & 3 & $(17,22,30)$ & 0.88 & $0.79,0.98$ & 0 & 0.38 \\
\hline Non-Asian & 4 & $(10,18,19,29)$ & 0.84 & $0.68,1.04$ & 81.9 & 0.001 \\
\hline \multicolumn{7}{|l|}{ Sex } \\
\hline Female & 3 & $(19,22,29)$ & 0.93 & $0.86,1.00$ & $27 \cdot 4$ & 0.001 \\
\hline Both & 4 & $(10,17,18,30)$ & 0.81 & $0.64,1.02$ & 53.8 & 0.029 \\
\hline \multicolumn{7}{|l|}{ Outcome assessment tools } \\
\hline CES-D & 4 & $(10,17,19,29)$ & 0.81 & $0.65,1.17$ & $82 \cdot 5$ & 0.001 \\
\hline Other tools & 3 & $(18,22,30)$ & 0.89 & $0.80,0.92$ & 0 & 0.45 \\
\hline \multicolumn{7}{|l|}{ Study quality } \\
\hline High quality (score $\geq 5$ ) & 6 & $(10,17-19,29,30)$ & 0.85 & $0.71,1.02$ & 73.1 & 0.002 \\
\hline Low quality $($ score $<5)$ & 1 & (22) & 0.88 & $0.79,0.97$ & _- & - \\
\hline Cross-sectional study & 8 & $(5,16,21,25,28,43)$ & 0.75 & $0.62,0.91$ & $56 \cdot 8$ & 0.023 \\
\hline \multicolumn{7}{|l|}{ Asian $v$. non-Asian } \\
\hline Asian & 6 & $(21,25,28,43)$ & 0.62 & $0.50,0.76$ & 0 & 0.50 \\
\hline Non-Asian & 2 & $(5,16)$ & 0.92 & $0.84,1.00$ & 0 & 0.71 \\
\hline \multicolumn{7}{|l|}{ Dietary assessment tools } \\
\hline $\mathrm{FFQ}$ & 3 & $(5,16,21)$ & 0.87 & $0.75,1.02$ & $48 \cdot 9$ & 0.14 \\
\hline Other tools & 5 & $(25,28,43)$ & 0.62 & $0.47,0.80$ & 7.5 & 0.36 \\
\hline \multicolumn{7}{|l|}{ Outcome assessment tools } \\
\hline GDS & 3 & $(21,25,43)$ & 0.54 & $0.41,0.72$ & 0 & 0.39 \\
\hline Other tools & 5 & $(5,16,28)$ & 0.90 & $0.83,0.98$ & 0 & 0.63 \\
\hline \multicolumn{7}{|l|}{ Energy adjustment } \\
\hline Adjusted & 7 & $(5,16,25,28,43)$ & 0.78 & $0.64,0.95$ & 54.8 & 0.039 \\
\hline Non-adjusted & 1 & (21) & 0.63 & $0.44,0.92$ & - & - \\
\hline \multicolumn{7}{|l|}{ Study quality } \\
\hline High quality (score $\geq 5$ ) & 5 & $(5,25,28)$ & 0.74 & $0.56,0.97$ & 57.9 & 0.050 \\
\hline Low quality $($ score $<5)$ & 3 & $(16,21,43)$ & 0.71 & $0.45,1.11$ & 69.8 & 0.036 \\
\hline
\end{tabular}

CES-D, Center for Epidemiological Studies-Depression Scale SF-36; Geriatric Depression Scale.

the year $2030^{(52)}$. Several investigations have suggested that enhanced oxidative stress or defective antioxidant defenses may be related to psychiatric disorders ${ }^{(25)}$. Recent investigations have suggested a link between dietary intakes and mental health. Increased intake of fruit and vegetables might be an important global dietary strategy to promote brain function and reduce non-communicable disease ${ }^{(47)}$.
We found an inverse association between fruit, vegetables and total fruit and vegetable intake and the risk of depression. These findings were in line with a previous meta-analysis on ten $\operatorname{articles}^{(24)}$. However, the findings of the previous meta-analysis might be distorted ${ }^{(55)}$, owing to the lack of considering several published studies in the field ${ }^{(10,14,25,26)}$ and using inappropriate statistical methods ${ }^{(5)}$. In the present meta-analysis, all these 


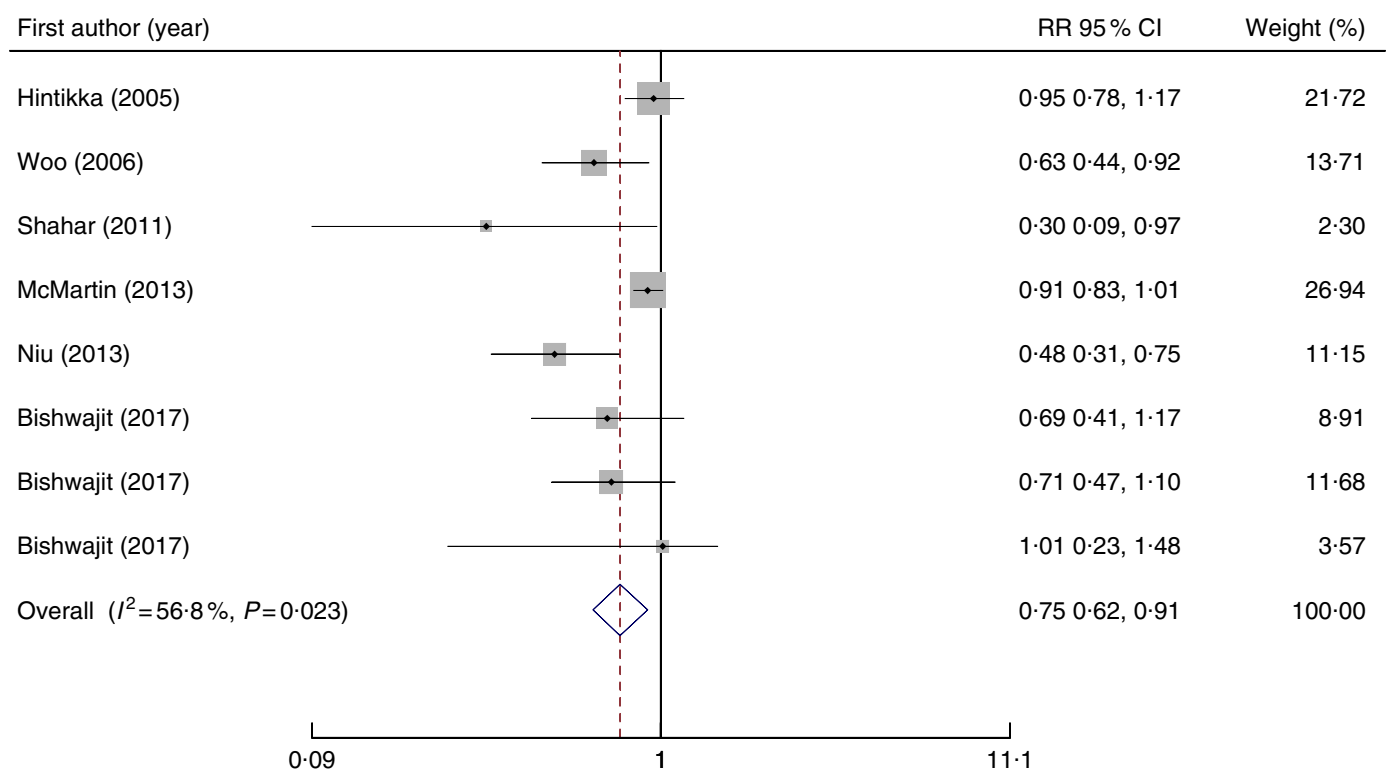

Fig. 6. Forest plots of the association between consumption of vegetables and depression in cross-sectional studies. RR, relative risk.

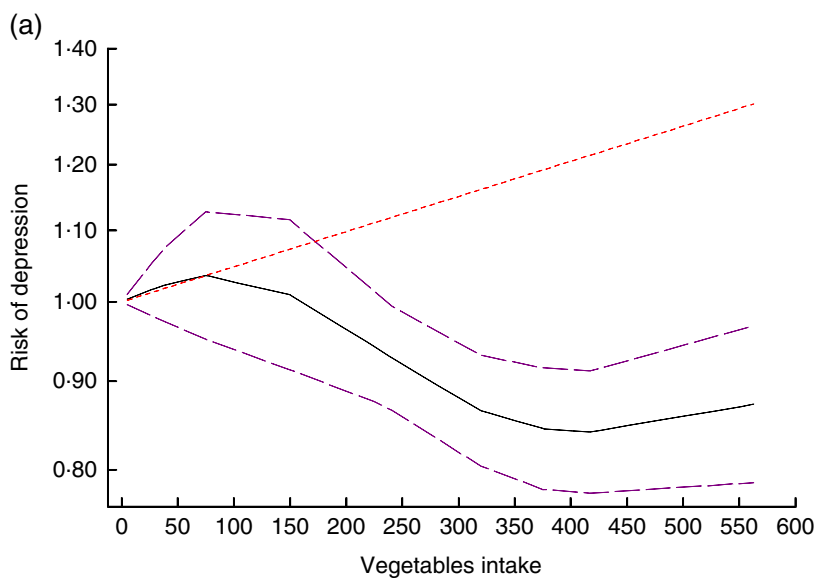

(b)

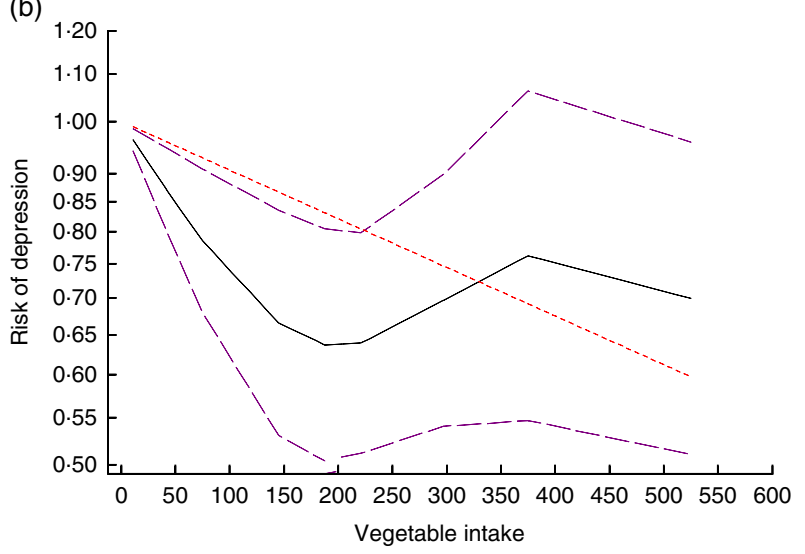

Fig. 7. Dose-response association between consumption of vegetables and risk of depression in cohort (a) and cross-sectional (b) studies. - - - - -, Linear model; $\_$, spline model.

points were taken into account. Findings from meta-analyses on the association of dietary patterns and depression have indicated that healthy dietary patterns, greatly loaded with fruit and vegetables, were associated with decreased odds of depression ${ }^{(9)}$. This was also confirmed by another systematic review on observational studies ${ }^{(56)}$. One of the important issues in this regard was that whether it is total fruit and vegetable intake that protects against psychological disorders, or fruit or vegetable consumption per $s e^{(57)}$. In the present study, we found that both fruit and vegetable intake, as well as their combination, could protect against the risk of depression in cohort or crosssectional studies. Only one case-control study ${ }^{(40)}$ had reported OR of the association between vegetable intake and risk of depression, which we did not include in the present analysis owing to several biases in case-control studies. However, combining this study ${ }^{(40)}$ with cross-sectional studies did not result in significant changes in our findings (overall $\mathrm{RR}=0 \cdot 80 ; 95 \% \mathrm{CI} 0.64$, $\left.0 \cdot 99, I^{2}=68.4 \%, P=0 \cdot 001\right)$. Quality of studies could be one of the reasons for the various results observed in previous studies. Highquality studies $^{(5,17-19,25,28-30,54)}$ have reported greater reduction in the risk of depression compared with low-quality studies.

Fruit and vegetables are rich sources of fibre, vitamins and minerals, antioxidants, flavonoids and phytochemicals. This nutrient content has protective effects against depression. In biological systems, some dietary nutrients such as $\beta$-carotenes, vitamin $\mathrm{E}$ and vitamin $\mathrm{C}$ have been reported as effective antioxidants. Oxidative stress, defined as a disturbance in the balance between the production of reactive oxygen species (free radicals) and antioxidant defenses ${ }^{(58-60)}$, is thought to contribute to the incidence of depression. Nutrient deficiency might be another plausible pathway for the linkage between fruit and vegetable intake with depression. A deficiency in either folate, which is involved in neurotransmitter synthesis, or vitamin $B_{12}$ could elevate homocysteine levels and increase the risk of depression ${ }^{(61)}$. Moreover, pyridoxal phosphate, an active form of vitamin $\mathrm{B}_{6}$, is involved in the synthesis of neurotransmitters, such as serotonin, and elevating mood ${ }^{(62)}$.

This study has some strengths and limitations. Obtaining the overall effect using meta-analysis, applying subgroup analysis 


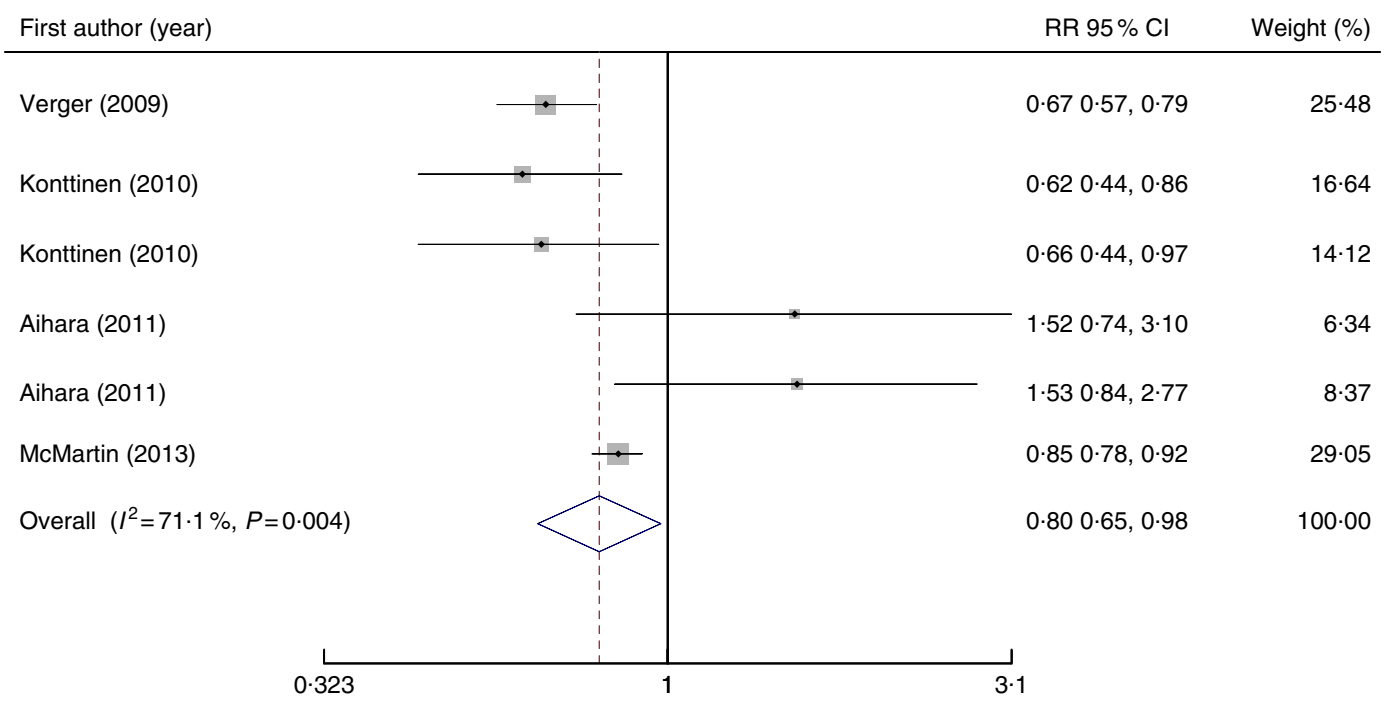

Fig. 8. Forest plots of the association between total intake of fruits and vegetables and risk of depression. RR, relative risk.

Table 5. Results of subgroup analysis for total intake of fruit and vegetables and risk of depression (Odds ratios and $95 \%$ confidence intervals)

\begin{tabular}{|c|c|c|c|c|c|c|}
\hline & No. of effect sizes & Ref. & OR & $95 \% \mathrm{Cl}$ & $I^{2}(\%)$ & $P_{\text {Heterogeneity }}$ \\
\hline Overall & 6 & $(5,14,26,27)$ & 0.80 & $0.65,0.98$ & $71 \cdot 1$ & 0.004 \\
\hline \multicolumn{7}{|l|}{ Asian $v$. non-Asian } \\
\hline Asian & 2 & $(27)$ & 1.53 & $0.96,2.41$ & 0 & 0.99 \\
\hline Non-Asian & 4 & $(5,14,26)$ & 0.72 & $0.60,0.87$ & $69 \cdot 1$ & 0.021 \\
\hline \multicolumn{7}{|l|}{ Sex } \\
\hline Male & 2 & $(14,27)$ & 0.95 & $0.42,2 \cdot 13$ & $75 \cdot 1$ & 0.046 \\
\hline Female & 2 & $(14,27)$ & 0.94 & $0.39,2.27$ & $85 \cdot 1$ & 0.010 \\
\hline Both & 2 & $(5,26)$ & 0.76 & $0.60,0.96$ & $84 \cdot 6$ & 0.011 \\
\hline \multicolumn{7}{|l|}{ Dietary assessment tools } \\
\hline $\mathrm{FFQ}$ & 4 & $(5,14,26)$ & 0.72 & $0.60,0.87$ & $69 \cdot 1$ & 0.021 \\
\hline MAFF questionnaire & 2 & (27) & 1.53 & $0.96,2.41$ & 0 & 0.99 \\
\hline \multicolumn{7}{|l|}{ Outcome assessment tools } \\
\hline CES-D & 3 & $(14,26)$ & 0.66 & $0.57,0.76$ & 0 & 0.92 \\
\hline GDS & 2 & (27) & 1.53 & $0.96,2.41$ & 0 & 0.99 \\
\hline CIDI & 1 & (5) & 0.85 & $0.78,0.92$ & - & - \\
\hline \multicolumn{7}{|l|}{ Study quality } \\
\hline High quality (score $\geq 5$ ) & 5 & $(5,14,27)$ & 0.86 & $0.66,1.12$ & $64 \cdot 2$ & 0.025 \\
\hline Low quality (score $<5$ ) & 1 & $(26)$ & 0.67 & $0.57,0.79$ & - & - \\
\hline
\end{tabular}

MAFF, Ministry of Agriculture, Forestry and Fishery; CES-D, Center for Epidemiological Studies-Depression Scale; GDS, Geriatric Depression Scale; CIDI, Composite International Diagnostic Interview.

to find the source of heterogeneity and using the estimates with a maximum adjustment were among the strengths of the study. The first limitation of our study was seasonal changes in depressive symptoms; these variations might affect the results of the included investigations ${ }^{(17)}$. Another limitation was using different dietary assessment tools in different studies to measure fruit and vegetable intake. This might potentially influence the associations. Dietary recall has higher precision in assessing dietary intakes but measures actual intake and cannot reflect the long-term usual intakes of the population. FFQ, on the other hand, measures long-term usual intakes, but it is subject to many errors introduced as a result of restrictions to a fixed list of foods, memory and perception of portion sizes, which can lead to misclassification of study participants ${ }^{(9)}$. Furthermore, different diagnostic criteria were used for defining depression in different studies. Although most applied questionnaires were valid tools, using different ways of scoring and cut-off points to define depression might affect the results ${ }^{(9)}$. The inconsistent adjustment for potential confounders among the included studies might also contribute to between-study heterogeneity. We extracted the RR with a maximum adjustment for potential confounders; however, the extent to which these estimates were adjusted and the residual confounding by other unmeasured factors should be considered. Finally, few studies were available investigating fruit and vegetable intake and anxiety relations. More investigations in this regard are needed to draw a conclusion.

This meta-analysis of observational studies provides further evidence that fruit and vegetables intake was protectively associated with depression. The findings support the current recommendation of increasing fruit and vegetable intake to improve mental health. 


\section{Acknowledgements}

This study was financially supported by a joint collaboration of Endocrinology and Metabolism Molecular-Cellular Sciences Institute, Tehran University of Medical Sciences, and School of Nutritional Sciences and Dietetics, Tehran University of Medical Sciences, Tehran, Iran. Dr A. E. was supported by a grant from Iran National Science Foundation.

F. S., H. M., P. S., A. M., B. L. and A. E. contributed to conception, design, statistical analyses, data interpretation and manuscript drafting. All authors approved the final manuscript for submission.

The authors declare that there are no conflicts of interest.

\section{References}

1. Jacka FN, Mykletun A \& Berk M (2012) Moving towards a population health approach to the primary prevention of common mental disorders. BMC Med 10, 149.

2. Chocano-Bedoya PO, O'Reilly EJ, Lucas M, et al. (2013) Prospective study on long-term dietary patterns and incident depression in middle-aged and older women. Am J Clin Nutr 98, 813-820.

3. Prohan M, Amani R, Nematpour S, et al. (2014) Total antioxidant capacity of diet and serum, dietary antioxidant vitamins intake, and serum hs-CRP levels in relation to depression scales in university male students. Redox Rep 19, 133-139.

4. Yannakoulia M, Panagiotakos DB, Pitsavos C, et al. (2008) Eating habits in relations to anxiety symptoms among apparently healthy adults. A pattern analysis from the ATTICA Study. Appetite 51, 519-525.

5. McMartin SE, Jacka FN \& Colman I (2013) The association between fruit and vegetable consumption and mental health disorders: evidence from five waves of a national survey of Canadians. Prev Med 56, 225-230.

6. Bhattacharyya M, Marston L, Walters K, et al. (2014) Psychological distress, gender and dietary factors in South Asians: a cross-sectional survey. Public Health Nutr 17, 1538-1546.

7. Quirk SE, Williams LJ, O'Neil A, et al. (2013) The association between diet quality, dietary patterns and depression in adults: a systematic review. BMC Psychiatry 13, 175.

8. Sanchez-Villegas A \& Martinez-Gonzalez MA (2013) Diet, a new target to prevent depression? BMC Med 11, 3 .

9. Lai JS, Hiles S, Bisquera A, et al. (2014) A systematic review and meta-analysis of dietary patterns and depression in community-dwelling adults. Am J Clin Nutr 99, 181-197.

10. Akbaraly TN, Sabia S, Shipley MJ, et al. (2013) Adherence to healthy dietary guidelines and future depressive symptoms: evidence for sex differentials in the Whitehall II study1. Am J Clin Nutr 97, 419-427.

11. Kuczmarski MF, Cremer Sees A, Hotchkiss L, et al. (2010) Higher Healthy Eating Index-2005 scores associated with reduced symptoms of depression in an urban population: findings from the Healthy Aging in Neighborhoods of Diversity Across the Life Span (HANDLS) study. J Am Diet Assoc 110, 383-389.

12. McMartin SE, Kuhle S, Colman I, et al. (2012) Diet quality and mental health in subsequent years among Canadian youth. Public Health Nutr 15, 2253-2258.

13. Khalid S, Williams CM \& Reynolds SA (2017) Is there an association between diet and depression in children and adolescents? A systematic review. Br J Nutr 116, 2097-2108.

14. Konttinen H, Mannisto S, Sarlio-Lahteenkorva S, et al. (2010) Emotional eating, depressive symptoms and self-reported food consumption. A population-based study. Appetite 54, 473-479.
15. Kim TH, Choi JY, Lee HH, et al. (2015) Associations between Dietary Pattern and Depression in Korean Adolescent Girls. J Pediatr Adolesc Gynecol 28, 533-537.

16. Hintikka J, Tolmunen T, Honkalampi K, et al. (2005) Daily tea drinking is associated with a low level of depressive symptoms in the Finnish general population. Eur J Epidemiol 20, 359-363.

17. Chi SH, Wang JY \& Tsai AC (2015) Combined association of leisure-time physical activity and fruit and vegetable consumption with depressive symptoms in older Taiwanese: Results of a national cohort study. Geriatr Gerontol Int 16, 244-251.

18. Sanchez-Villegas A, Delgado-Rodriguez M, Alonso A, et al. (2009) Association of the Mediterranean dietary pattern with the incidence of depression: the Seguimiento Universidad de Navarra/University of Navarra follow-up (SUN) cohort. Arch Gen Psychiatry 66, 1090-1098.

19. Mihrshahi S, Dobson AJ \& Mishra GD (2015) Fruit and vegetable consumption and prevalence and incidence of depressive symptoms in mid-age women: results from the Australian longitudinal study on women's health. Eur J Clin Nutr 69, 585-591.

20. Tsai AC, Chang TL \& Chi SH (2012) Frequent consumption of vegetables predicts lower risk of depression in older Taiwanese - results of a prospective population-based study. Public Health Nutr 15, 1087-1092.

21. Woo J, Lynn H, Lau WY, et al. (2006) Nutrient intake and psychological health in an elderly Chinese population. Int J Geriatr Psychiatry 21, 1036-1043.

22. Gangwisch JE, Hale L, Garcia L, et al. (2015) High glycemic index diet as a risk factor for depression: analyses from the Women's Health Initiative. Am J Clin Nutr 102, 454-463.

23. Allgöwer A, Wardle J \& Steptoe A (2001) Depressive symptoms, social support, and personal health behaviors in young men and women. Health Psychol 20, 223-227.

24. Liu X, Yan Y, Li F, et al. (2015) Fruit and vegetable consumption and the risk of depression: a meta-analysis. Nutrition 32, 296-302.

25. Niu K, Guo H, Kakizaki M, et al. (2013) A tomato-rich diet is related to depressive symptoms among an elderly population aged 70 years and over: a population-based, cross-sectional analysis. J Affect Disord 144, 165-170.

26. Verger P, Lions C \& Ventelou B (2009) Is depression associated with health risk-related behaviour clusters in adults? Eur J Public Health 19, 618-624.

27. Aihara Y, Minai J, Aoyama A, et al. (2011) Depressive symptoms and past lifestyle among Japanese elderly people. Community Ment Health J 47, 186-193.

28. Bishwajit G, O'Leary DP, Ghosh S, et al. (2017) Association between depression and fruit and vegetable consumption among adults in South Asia. BMC Psychiatry 17, 15.

29. Rienks J, Dobson AJ \& Mishra GD (2013) Mediterranean dietary pattern and prevalence and incidence of depressive symptoms in mid-aged women: results from a large community-based prospective study. Eur J Clin Nutr 67, 75-82.

30. Woo J, Ho SC \& Yu ALM (2002) Lifestyle factors and health outcomes in elderly Hong Kong Chinese aged 70 years and over. Gerontology 48, 234-240.

31. Dressler H \& Smith C (2015) Depression affects emotional eating and dietary intake and is related to food insecurity in a group of multiethnic, low-income women. J Hunger Environmental Nutr 10, 496-510.

32. Kulkarni RS \& Shinde RL (2015) Depression and its associated factors in older Indians: a study based on Study of Global Aging and Adult Health (SAGE)-2007. J Aging Health 27, 622-649. 
33. Poorrezaeian M, Siassi F, Qorbani M, et al. (2015) Association of dietary diversity score with anxiety in women. Psychiatry Res 230, 622-627.

34. Chang MW, Brown R, Nitzke S, et al. (2015) Stress, sleep, depression and dietary intakes among low-income overweight and obese pregnant women. Matern Child Health $J \mathbf{1 9}$, 1047-1059.

35. Chatzi L, Melaki V, Sarri K, et al. (2011) Dietary patterns during pregnancy and the risk of postpartum depression: the motherchild 'Rhea' cohort in Crete, Greece. Public Health Nutr 14, $1663-1670$.

36. Robinson M, Kendall GE, Jacoby P, et al. (2011) Lifestyle and demographic correlates of poor mental health in early adolescence. I Paediatr Child Health 47, 54-61.

37. Kohlboeck G, Sausenthaler S, Standl M, et al. (2012) Food intake, diet quality and behavioral problems in children: results from the GINI-plus/LISA-plus studies. Ann Nutr Metab 60, 247-256.

38. Oddy WH, Robinson M, Ambrosini GL, et al. (2009) The association between dietary patterns and mental health in early adolescence. Prev Med 49, 39-44.

39. Renzaho AM, Kumanyika S \& Tucker KL (2011) Family functioning, parental psychological distress, child behavioural problems, socio-economic disadvantage and fruit and vegetable consumption among 4-12 year-old Victorians, Australia. Health Promot Int 26, 263-275.

40. Michalak J, Zhang XC \& Jacobi F (2012) Vegetarian diet and mental disorders: results from a representative community survey. Int J Behav Nutr Phys Act 9, 67.

41. Wells GA OCD, Peterson J, Welch V, et al., The NewcastleOttawa Scale (NOS) for assessing the quality of nonrandomised studies in meta-analyses. http://www.ohri.ca/programs/ clinical_epidemiology/oxford.htm (accessed June 2017).

42. Liu C, Xie B, Chou CP, et al. (2007) Perceived stress, depression and food consumption frequency in the college students of China Seven Cities. Physiol Behav 92, 748-754.

43. Shahar S, Hassan J, Sundar VV, et al. (2011) Determinants of depression and insomnia among institutionalized elderly people in Malaysia. Asian J Psychiatr 4, 188-195.

44. Orsini N, Bellocco R \& Greenland S (2006) Generalized least squares for trend estimation of summarized dose-response data. Stata J 6, 40-57.

45. Whitaker KM, Sharpe PA, Wilcox S, et al. (2014) Depressive symptoms are associated with dietary intake but not physical activity among overweight and obese women from disadvantaged neighborhoods. Nutr Res 34, 294-301.

46. El Ansari W, Adetunji H \& Oskrochi R (2014) Food and mental health: relationship between food and perceived stress and depressive symptoms among university students in the United Kingdom. Cent Eur J Public Health 22, 90-97.
47. Crichton GE, Bryan J, Hodgson JM, et al. (2013) Mediterranean diet adherence and self-reported psychological functioning in an Australian sample. Appetite 70, 53-59.

48. Forsyth AK, Williams PG \& Deane FP (2012) Nutrition status of primary care patients with depression and anxiety. Aust J Prim Health 18, 172-176.

49. Castellanos D, Connell C \& Lee J (2011) Factors affecting weight gain and dietary intake in Latino males residing in Mississippi: a preliminary study. Hispanic Health Care Int 9, 91-98.

50. Mikolajczyk RT, El Ansari W \& Maxwell AE (2009) Food consumption frequency and perceived stress and depressive symptoms among students in three European countries. Nutr J $\mathbf{8}, 31$.

51. Kingsbury M, Dupuis G, Jacka F, et al. (2016) Associations between fruit and vegetable consumption and depressive symptoms: evidence from a national Canadian longitudinal survey. I Epidemiol Community Health 70, 155-161.

52. Payne ME, Steck SE, George RR, et al. (2012) Fruit, vegetable, and antioxidant intakes are lower in older adults with depression. J Acad Nutr Diet 112, 2022-2027.

53. Abdi S, Salehi N, Ghodsi B, et al. (2012) Immediate results of percutaneous trans-luminal mitral commissurotomy in pregnant women with severe mitral stenosis. Clin Med Insights: Cardiol 6, 35-39.

54. Akbaraly TN, Sabia S, Shipley MJ, et al. (2013) Adherence to healthy dietary guidelines and future depressive symptoms: evidence for sex differentials in the Whitehall II study. Am J Clin Nutr 97, 419-427.

55. Saneei P, Saghafian F \& Esmaillzadeh A (2016) Meta-analysis on the association of fruit and vegetable intake with depression: further analysis is required. Nutrition 32, 1162-1163.

56. Rahe C, Unrath M \& Berger K (2014) Dietary patterns and the risk of depression in adults: a systematic review of observational studies. Eur J Nutr 53, 997-1013.

57. Hu D, Huang J, Wang Y, et al. (2014) Fruits and vegetables consumption and risk of stroke: a meta-analysis of prospective cohort studies. Stroke 45, 1613-1619.

58. Betteridge DJ (2000) What is oxidative stress? Metabolism 49, 3-8.

59. Birben E, Sahiner UM, Sackesen C, et al. (2012) Oxidative stress and antioxidant defense. World Allergy Organ J 5, 9-19.

60. Burton GJ \& Jauniaux E (2011) Oxidative stress. Best Pract Res Clin Obstet Gynaecol 25, 287-299.

61. Kim J-M, Stewart R, Kim S-W, et al. (2008) Predictive value of folate, vitamin $\mathrm{B}_{12}$ and homocysteine levels in late-life depression. Br J Psychiatry 192, 268-274.

62. Williams A-l, Cotter A, Sabina A, et al. (2005) The role for vitamin B-6 as treatment for depression: a systematic review. Fam Pract 22, 532-537. 